\title{
Teor e qualidade de substâncias húmicas de planossolo sob diferentes sistemas de cultivo
}

\author{
Content and quality of humic substances of a Albaqualf under different management systems
}

\author{
Carla Machado da RosaI Rosa Maria Vargas Castilhos"II Deborah Pinheiro Dick ${ }^{\mathrm{III}}$ \\ Eloy Antonio Pauletto ${ }^{\text {II }}$ Algenor da Silva Gomes ${ }^{\text {IV }}$
}

RESUMO

Este trabalho teve por objetivo avaliar e comparar os teores e as características químicas das substâncias húmicas (SHs) de um Planossolo háplico submetido a diferentes sistemas de cultivos de arroz. Amostras de solo foram coletadas em um experimento de longa duração (19 anos) nas profundidades de 0-0,025, 0,025-0,05, 0,05-0,20m e no topo do horizonte $B$. Os tratamentos avaliados foram: APC-Sistema de cultivo contínuo de arroz (preparo convencional) e controle de invasoras com herbicida, APD-Sucessão de azevém x arroz sob plantio direto e SN-Solo mantido em condições naturais (pastagem nativa). As SHs e a fração não-húmica (NH) foram obtidas por meio de fracionamento químico. O teor de carbono do solo e na forma de SHs e NH foram determinados, respectivamente, pelos métodos Walkley-Black e espectrofotométrico. As características químicas e moleculares das $\mathrm{SHs}$ foram avaliadas por espectroscopias de infravermelho e UV/Vis e por análise elementar. Em comparação ao solo natural, o sistema APD preservou os teores de carbono orgânico total e de todas as frações húmicas, enquanto que o sistema APC provocou perdas de carbono orgânico total e de todas as frações húmicas nas camadas superficiais $(<0,05 m)$. A proporção de ácidos fúlvicos em relação ao carbono total aumentou em profundidade, sugerindo mobilização desta fração no perfil. O sistema de manejo afetou a composição da matéria orgânica principalmente na camada de 0 a 0,025m, ocorrendo SHs de menor grau de decomposição, mais polares e contendo menor proporção de estruturas alifáticas que no sistema APD.

Palavras-chave: solo de várzea, matéria orgânica, arroz irrigado, experimento longa duração.

\begin{abstract}
The objective of this study was to evaluate and compare the content and chemical characteristics of humic substances (SHs) of an Albaqualf under different soil management systems. Soil samples were collected in a longterm field experiment (19 years) from the 0-0.025; 0.025-0.05 and $0.05-0.20 m$ layers and from the top of the $B$ horizon. The following treatments were evaluated: APC-continuous rice crop system under conventional tillage and weed control with herbicides; APD-no-tillage system with rye-grass in the winter and rice in the summer, and $\mathrm{SN}$-soil under natural condition with native grassland. The SHs and the non-humic fraction (NH) were obtained by chemical fractioning. The content of total soil carbon and those occurring as SHs and $\mathrm{NH}$ were determined by the Walkley-Black and spectrophotometric methods, respectively. To determine the chemical and molecular characteristics of the SHs, UV-visible and infrared spectroscopy techniques and elementar analyses were employed. In comparison to the soil under natural condition (SN), the notillage system (APD) maintained total soil organic carbon concentrations as well as the content of SHs. The conventional tillage reduced soil total organic carbon and the content of $\mathrm{SH}$ in the upper layers $(<0.05 \mathrm{~m})$. The proportion of fulvic acid in relation to the total soil organic carbon increased with soil depth, suggesting the mobilization of this fraction along the profile. The soil management system affected the composition of the organic matter mainly in the 0 to $0.025 m$ layer, where the SH showed a lower decomposition and higher polaricity degree and contained smaller proportion of aliphatic structures than $\mathrm{SH}$ in the APD system.
\end{abstract}

Key words: lowlands, organic matter, irrigated rice, longterm field experiment.

IPrograma de Pós-graduação em Agronomia, Área de concentração em Solos, Universidade Federal de Pelotas (UFPel), CP 354, 96010-900, Pelotas, RS, Brasil. E-mail: carlamrosa@yahoo.com.br. Autor para correspondência

IDepartamento de Solos, Faculdade de Agronomia “Eliseu Maciel” (FAEM), UFPel, Pelotas, RS, Brasil.

IIIInstituto de Química, Universidade Federal do Rio Grande do Sul (UFRGS), Porto Alegre, RS, Brasil.

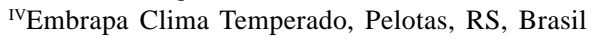




\section{INTRODUÇÃO}

A região Sul do Brasil é a principal produtora de arroz, respondendo por mais da metade (63\%) da produção nacional deste grão (AZAMBUJA et al., 2004). No Estado do Rio Grande do Sul (RS), existem aproximadamente 5,4 milhões de hectares de solos de várzea, dos quais aproximadamente um milhão de hectares é cultivado com arroz irrigado por inundação (PINTO et al., 2004).

De acordo com os dados da safra 2004/2005, entre os sistemas de cultivo utilizados para o arroz irrigado no RS, os sistemas conservacionistas (cultivo mínimo e plantio direto) têm aumentando significativamente sua participação (67\%) em relação à área total semeada, em comparação ao sistema convencional (22\%) (OLIVEIRA, 2006).

Os solos de várzea diferem dos solos de terras altas por estarem submetidos à condição de má drenagem e/ou sob alagamento durante o cultivo do arroz, fato que determina uma dinâmica diferenciada para as transformações da matéria orgânica do solo. Nestas condições de escassez de oxigênio, a decomposição da matéria orgânica (MO) se dá mais lentamente, prevalecendo os processos anaeróbios (TAN, 2003). Entretanto, ao final do ciclo do arroz, as lavouras são drenadas, prevalecendo o processo aeróbio.

A estabilidade da MO é afetada pela redução do solo sob condições de alagamento. MAIE et al. (1998), ao analisarem o efeito da redução do solo sobre a estabilidade do húmus, concluíram que a redução do ferro estava associada às mudanças no tipo de ligação do húmus. OLK et al. (2000), ao avaliarem os efeitos da colheita contínua de arroz sobre as características químicas da $\mathrm{MO}$, verificaram que com o aumento do alagamento do solo, as frações de ácido húmico tornaram-se menos humificadas, com altas concentrações de hidrogênio e baixas de oxigênio, mais grupos amida e amina, hidroxila e metoxila, menos grupos carboxila e radicais orgânicos livres. KIRK \& OLK (2000) constataram que, com aumento da submersão do solo, as frações de ácidos húmicos tornaram-se menos oxidadas ou humificadas.

Análises espectroscópicas têm sido utilizadas nos estudos de substâncias húmicas para determinar o grau de humificação e condensação das moléculas húmicas, bem como identificar grupos funcionais (ácidos carboxílicos, aminas, amidas, estruturas alifáticas e aromáticas, etc) (PAIM et al., 1990).

Vários estudos têm sido realizados no intuito de caracterizar a MO dos solos e suas alterações em decorrência dos diferentes sistemas de cultivo (BAYER, 1999; ALMEIDA, 2005; COSTA, 2004). Amaioria destes, entretanto, foi realizada em solos bem drenados, existindo uma carência de estudos com solos de várzea. Assim, o presente trabalho teve por objetivo avaliar, em experimento de longa duração, as diferenças no teor e na qualidade da matéria orgânica de um Planossolo háplico decorrentes da utilização de diferentes sistemas de cultivo, com respeito ao teor de substâncias húmicas e às suas características químicas e moleculares.

\section{MATERIAL E MÉTODOS}

O experimento foi implantado, em 1985, na Estação Experimental de Terras Baixas - CPACTEMBRAPA, localizado no município do Capão do Leão,

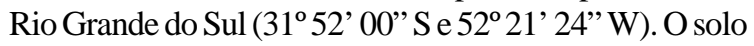
é um Planossolo háplico eutrófico gleico, com argila de atividade alta e relevo plano, tendo como substrato sedimentos derivados de granito (EMBRAPA, 1999). Os tratamentos estão dispostos em blocos casualizados com quatro repetições e são: APC-Sistema de cultivo contínuo de arroz (preparo convencional), APDSucessão de azevém x arroz sob plantio direto e SNSolo mantido com vegetação natural (predomíneo de gramíneas). Amostras de solo foram coletadas em outubro de 2004 (com solo seco), nas profundidades: 0-0,025; 0,025-0,05; 0,05-0,20m e topo do horizonte B (profundidade variando de 0,20 a $0,60 \mathrm{~m}$ ). As amostras foram secas ao ar, moídas e peneiradas em peneira de $2 \mathrm{~mm}$.

As substâncias húmicas foram fracionadas quimicamente, segundo DICK et al. (1998). Em 8g de solo foram adicionados $30 \mathrm{~mL}$ de $\mathrm{HCl} 0,5 \mathrm{~mol} \mathrm{~L}^{-1}$ e a suspensão foi agitada por duas horas. Após centrifugação (2500rpm, 15min), o sobrenadante foi separado e este procedimento foi repetido mais uma vez. Este extrato ácido (substâncias orgânicas nãohúmicas (NH)) teve seu volume medido e armazenado. Em seguida, o solo foi submetido a tratamento com $30 \mathrm{~mL}$ de $\mathrm{NaOH} 0,5 \mathrm{~mol} \mathrm{~L}^{-1}$ sob agitação (três horas). $\mathrm{O}$ extrato alcalino foi separado por centrifugação (2500rpm, 15min). Este procedimento foi repetido até o sobrenadante tornar-se incolor. Os extratos alcalinos correspondentes às substâncias húmicas solúveis (SHs) foram armazenados e o volume final foi medido e uma alíquota de $5 \mathrm{~mL}$ foi retirada para análises posteriores.

O extrato de SHs foi acidificado a $\mathrm{pH} 2,0$ com $\mathrm{HCl} 0,1 \mathrm{~mol} \mathrm{~L}{ }^{-1}$. Após 24 horas de repouso, a fração de ácidos fúlvicos (AF) (sobrenadante) foi separada por centrifugação do material precipitado correspondente a fração ácido húmico (AH). 
O carbono orgânico total do solo (COT) foi determinado pelo método Walkley-Black modificado, baseado na oxidação do carbono orgânico pelo dicromato de potássio, sem aquecimento. Os teores de $\mathrm{C}$ no extrato ácido (CNH), nas SHs (CSHs) e no AF (CAF) foram quantificados, determinando-se a absorbância a 580nm após oxidação da MO com dicromato de potássio em meio ácido a $60^{\circ} \mathrm{C}$, durante quatro horas (DICK et al., 1998). O teor de C na forma de $\mathrm{AH}$ e na forma de HU foram determinados pelas diferenças $\mathrm{CAH}=\mathrm{CSH}$ - CAF e CHU = COT - CNH CSHs, respectivamente.

Foram determinadas, em espectrômetro UVVis, as absorbâncias em 465 (E4) e em 665nm (E6) dos sobrenadantes de SHse de AF, calculando-se a razão E4/E6 (CHEN et al., 1997). Para poder comparar os resultados das duas frações, a suspensão de AF foi alcalinizada a $\mathrm{pH} 12$, adicionando-se $\mathrm{NaOH} 0,1 \mathrm{~mol} \mathrm{~L}^{-1}$ previamente à análise. Considerando-se que as frações de $\mathrm{SH}$ e de $\mathrm{AF}$ não foram purificadas previamente à análise de UV/Vis, foram traçados os espectros de 200 a 800nm das soluções a fim de averiguar a interferência de componentes inorgânicos solubilizados. Não foram detectados picos de absorbância ao longo do espectro, e, portanto, considerou-se a ausência de complexos metal-orgânicos que pudessem interferir sobre a determinação de E4/E6. Para as demais análises qualitativas, os $\mathrm{AH}$ (amostras compostas) foram purificados com 30mL de solução de $\mathrm{HF} / \mathrm{HCl}(5 \% \mathrm{v} / \mathrm{v})$ sob agitação por duas horas, repetindo-se o procedimento duas vezes (DICK et al., 1998). O material precipitado foi congelado com água destilada por uma noite e após descongelamento foi lavado com água destilada até teste negativo com $\mathrm{AgNO}_{3}$ para íons cloreto. $\mathrm{OAH}$ foi seco em estufa a $45^{\circ} \mathrm{C}$ e acondicionado em frascos com tampa.

Afim de comparar extremos do perfil, amostras de $\mathrm{AH}$, nas profundidades de 0-0,025m e 0,05$0,20 \mathrm{~m}$, foram analisadas por espectroscopia de infravermelho com transformada de Fourier (FTIR) em pastilhas de $\mathrm{KBr}$ (razão de 1:100). O espectro foi traçado (Shimadzu 830 FTIR) de 4000 a $400 \mathrm{~cm}^{-1}$, empregando-se resolução de $4 \mathrm{~cm}^{-1}$ e 32 scans min $^{-1}$. A partir dos espectros (em duplicata) foi calculado o índice I1630/I2920 (CHEFETZ et al., 1996), dividindo-se a intensidade de absorção em torno de $1630 \mathrm{~cm}^{-1}$ pela intensidade a $2922 \mathrm{~cm}^{-1}$. A composição elementar (teores de $\mathrm{C}, \mathrm{H}$ e N) dos AH foi determinada por combustão seca (Perkin Elmer 2400). O teor de oxigênio foi calculado por diferença após correção dos valores para uma base livre de cinzas: $\mathrm{O}(\%)=100-(\mathrm{C}+\mathrm{H}+\mathrm{N})$ corrigidos. O teor de cinzas foi determinado gravimetricamente, após calcinação a $750^{\circ} \mathrm{C}$, durante quatro horas (DICK et al., 1997). A partir destes dados foi calculada a relação $\mathrm{C} / \mathrm{N}$ e o índice de polaridade $(\mathrm{O}+\mathrm{N}) / \mathrm{C}$, considerando-se as proporções molares (TORRENTS et al, 1997).

Os resultados dos parâmetros avaliados foram submetidos à análise da variância e aplicado o teste de Duncan ao nível de 5\%, para avaliação da diferença entre as médias obtidas.

\section{RESULTADOS E DISCUSSÃO}

Os teores de carbono orgânico total (COT) variaram de 13,0 a 42,5g kg-1 na camada de $0-0,025 \mathrm{~m}$, de 11,6 a $15,5 \mathrm{~g} \mathrm{~kg}^{-1}$ na camada de $0,025-0,05 \mathrm{~m}$, de 7,8 a 9,7g $\mathrm{kg}^{-1}$ na camada de $0,05-0,20 \mathrm{~m}$ e de 5,8 a $6,5 \mathrm{~g} \mathrm{~kg}^{-1}$ no topo do horizonte B (Tabela 1). Nos três tratamentos, os valores e também a amplitude de variação diminuíram com a profundidade.

Na camada de $0-0,025 \mathrm{~m}$, os maiores teores de COT foram encontrados nos sistemas APD e SN, não havendo diferença entre eles. No SN, este fato pode ser explicado pela maior estabilidade deste sistema sem utilização agrícola, ocorrendo um equilíbrio dinâmico entre as taxas médias de adição de carbono no solo e as taxas de mineralização do resíduo orgânico. No APD, o não revolvimento do solo e o acúmulo dos resíduos orgânicos na camada superficial favoreceram o aumento do COT.

O tratamento APC apresentou redução nos teores de COT da camada de 0-0,025m quando comparadas aos sistemas APD e SN (Tabela 1). Esta diminuição foi de $69 \%$ e pode ser atribuída principalmente a maior taxa de mineralização da $\mathrm{MO}$ devido ao revolvimento do solo, pois a quebra dos agregados do solo no PC expõe a matéria orgânica à atividade microbiana, levando à perda de carbono na forma de $\mathrm{CO}_{2}$ (COSTA, 2004). Resultados semelhantes foram obtidos por SANTOS et al. (2004) na camada superficial (0-0,05m) de um solo de várzea cultivado com arroz irrigado, onde após 17 anos de cultivo, observou-se aumento de $47 \%$ no teor de COT no solo sob APD em relação ao preparo convencional.

$\mathrm{Na}$ camada superficial, os valores encontrados para os teores de carbono na forma de frações húmicas foram superiores e iguais nos sistemas SN e APD (Tabela 1). A distribuição do carbono nas frações humificadas da MO não foi alterada pelo cultivo do solo no sistema plantio direto em comparação com o solo não-cultivado. Em contrapartida, no sistema APC as reduções foram de $81 \%$ na fração HU, seguido de $69 \%$ na fração AF e de $48 \%$ na fração AH, em relação ao sistema SN.

Na camada de 0,025-0,05m, os sistemas de cultivo não diferiram do solo mantido sob condição natural (SN) quanto ao teor de carbono nas frações AF 
Tabela 1 - Teores de carbono orgânico total (COT) e na forma de ácido fúlvico (AF), de ácido húmico (AH) e de humina (HU) da matéria orgânica de um Planossolo háplico submetido a diferentes sistemas de cultivo, Capão do Leão - RS. Média de quatro repetições.

\begin{tabular}{|c|c|c|c|c|}
\hline \multirow{2}{*}{ Sistemas de cultivo } & $\mathrm{COT}$ & $\mathrm{AF}$ & $\mathrm{AH}$ & $\mathrm{HU}$ \\
\hline & \multicolumn{4}{|c|}{ - } \\
\hline & ------------- & 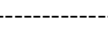 & 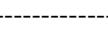 & ----------- \\
\hline APC & $13,01 \mathrm{~b}$ & $2,53 \mathrm{~b}$ & $5,47 \mathrm{~b}$ & $4,50 \mathrm{~b}$ \\
\hline APD & 39,07 a & $7,14 \mathrm{a}$ & 11,27 a & 19,38 a \\
\hline SN & $42,54 \mathrm{a}$ & 7,95 a & 10,45 a & 22,83 a \\
\hline APC & $11,64 \mathrm{~b}$ & $2,28 \mathrm{~b}$ & 5,60 & $3,26 b$ \\
\hline APD & 15,47 a & 3,24 a & 6,52 & $5,07 \mathrm{ab}$ \\
\hline SN & $14,72 \mathrm{ab}$ & $2,96 \mathrm{ab}$ & 4,72 & 6,43 a \\
\hline APC & 9,72 a & 2,27 a & 4,31 a & 2,68 \\
\hline APD & $8,54 \mathrm{ab}$ & $1,76 \mathrm{~b}$ & $3,19 \mathrm{~b}$ & 3,27 \\
\hline SN & $7,77 \mathrm{~b}$ & $1,87 \mathrm{~b}$ & $2,87 \mathrm{~b}$ & 2,59 \\
\hline APC & 5,78 & 1,79 & 1,84 & 1,82 \\
\hline APD & 6,27 & 1,70 & 1,69 & 2,62 \\
\hline SN & 6,51 & 1,83 & 1,72 & 2,59 \\
\hline
\end{tabular}

Médias seguidas da mesma letra na coluna não diferem estatisticamente pelo teste de Duncan a 5\% de probabilidade de erro. Ausência de letras indica que as médias não diferiram entre si.

APC = Sistema de cultivo contínuo de arroz (preparo convencional) e controle de invasoras com herbicida; APD = Sucessão de azevém x arroz sob plantio direto; $\mathrm{SN}$ = Solo mantido em condições naturais, sem cultivo.

e AH, evidenciando que as transformações da MO, com respeito ao teor de substâncias húmicas, em decorrência da utilização do solo com diferentes sistemas de cultivo, foram mais acentuadas na camada superficial $(0-0,025 \mathrm{~m})$. Entretanto, no tratamento APD, a quantidade de AF foi superior a do tratamento APC. O teor de carbono na forma de HU encontrado no sistema APD foi igual ao SN e o tratamento APC apresentou redução de 49\%, quando comparado ao solo mantido sob condição natural (Tabela 1). O preparo do solo pode ter contribuído para as perdas de carbono nas frações húmicas deste sistema.

Na profundidade de 0,05-0,20m, o sistemaAPC apresentou teores de COT superiores ao SN (Tabela 1). Isso se deve ao cultivo contínuo de arroz sob preparo convencional, em que a aração, além de incorporar os resíduos, homogeneíza os teores de carbono até esta profundidade. Além disso, este sistema, em comparação ao SN, deve apresentar uma taxa de decomposição mais lenta da MO, em razão da menor atividade microbiana ocasionada pelo ambiente alagado a que o solo é submetido anualmente, quando cultivado com arroz. O maior teor de carbono no sistema APC nesta profundidade se refletiu principalmente em enriquecimento das frações AF e AH, em comparação aos sistemas APD e SN.

No topo do horizonte B, os teores de COT foram inferiores às demais profundidades e não diferiram entre os tratamentos, o que pode ser atribuído ao fato de que este horizonte encontra-se abaixo da camada arável revolvida pelo preparo convencional do solo e, conseqüentemente é pouco afetado em sua estrutura e nos teores de COT. O mesmo comportamento foi observado com as frações húmicas.

Os valores de E4/E6 variaram entre 4,7 e 5,9 para as SHs (Tabela 2) e são semelhantes aos observados para AH de solos drenados (TAN, 2003). A elevada proporção de AH na fração de SHs (Tabela 1) estaria governando os valores desta razão, fazendo com que a mesma reflita principalmente as características dos $\mathrm{AH}$.

Na camada de 0-0,025m, os maiores valores da razão E4/E6 foram encontrados nos sistemas SN e $\mathrm{APD}$, indicando a presença de estruturas menos condensadas e/ou de estruturas micelares menores. Comportamento semelhante entre AH de Planossolo sob sistemas APD e APC em cultivo de arroz foi observado por OLENDZKI (2006). Provavelmente o não revolvimento do solo nesses sistemas e a permanência da palha de cobertura no APD conduzem a um acúmulo de frações orgânicas de menor grau de decomposição em relação ao APC. Neste sistema APC, em razão do revolvimento do solo, as frações orgânicas estariam mais sujeitas aos processos de humificação, ocorrendo um acúmulo de frações em grau de decomposição mais avançado.

Ciência Rural, v.38, n.6, set, 2008. 
Tabela 2 - Razão $\mathrm{E}_{4} / \mathrm{E}_{6}$ das substâncias húmicas solúveis (SHs) e do ácidos fúlvicos (AF) de um Planossolo háplico submetido a diferentes sistemas cultivo, Capão do Leão - RS. Média de quatro repetições.

\begin{tabular}{|c|c|c|c|c|}
\hline \multirow{2}{*}{ Sistemas de manejo } & \multicolumn{4}{|c|}{ 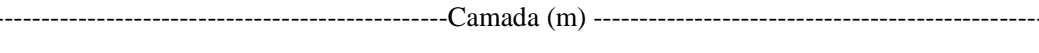 } \\
\hline & $0-0,025$ & $0,025-0,05$ & $0,05-0,20$ & topo do B \\
\hline & \multicolumn{4}{|c|}{-------------------------------------------------------- SHs ------------------------------------------------------- } \\
\hline APC & $5,03 \mathrm{~b}$ & 5,10 & 4,76 & 5,47 \\
\hline APD & $5,49 \mathrm{ab}$ & 5,62 & 4,88 & 5,29 \\
\hline SN & 5,73 а & 5,36 & 5,17 & 5,92 \\
\hline APC & $12,53 \mathrm{~b}$ & 13,35 & 11,73 & 8,80 \\
\hline APD & $12,61 \mathrm{~b}$ & 10,65 & 11,93 & 11,38 \\
\hline $\mathrm{SN}$ & 15,63 а & 13,65 & 12,36 & 12,26 \\
\hline
\end{tabular}

Médias seguidas da mesma letra na coluna não diferem estatisticamente pelo teste de Duncan a 5\% de probabilidade de erro. Ausência de letras indica que as médias não diferiram entre si.

APC = Sistema de cultivo contínuo de arroz (preparo convencional) e controle de invasoras com herbicida; APD = Sucessão de azevém $\mathrm{x}$ arroz sob plantio direto; $\mathrm{SN}$ = Solo mantido em condições naturais, sem cultivo.

Os valores desta razão para os AF foram superiores do que os das respectivas SHs (Tabela 2) indicando a presença de estruturas menores nesta fração, como era esperado. Para os AF, as diferenças entre os tratamentos também se restringiram à camada superficial (0-0,025m), onde o maior valor foi observado no SN. Não houve diferenças significativas nas razões E4/E6 das SHs e dos AF nas demais profundidades.

As principais regiões de absorção dos espectros de FTIR dos AH (Figura 1) e suas respectivas atribuições (TAN, 2003): estiramento de-OH de álcoois, fenóis e ácidos carboxílicos $\left(3385 \mathrm{~cm}^{-1}\right)$; estiramento simétrico e assimétrico de C-H de grupos alifáticos (2919 e $2850 \mathrm{~cm}^{-1}$ ); estiramento $\mathrm{C}=\mathrm{O}$ de ácidos carboxílicos e/ ou cetonas $\left(1715 \mathrm{~cm}^{-1}\right)$; ligação $\mathrm{C}=\mathrm{C}$ de anéis aromáticos e estiramento assimétrico -COO- $\left(1640 \mathrm{~cm}^{-1}\right)$; ligações peptídicas N-H ou estiramento $\mathrm{C}=\mathrm{C}$ aromático $\left(1538 \mathrm{~cm}^{-1}\right)$; deformação de C-H de grupos alifáticos (1457, 1414 e $1383 \mathrm{~cm}^{-1}$ ); estiramento -C-O e deformação O-H de ácidos carboxílicos $\left(1234 \mathrm{~cm}^{-1}\right)$; ligação C-O de polissacarídeos $\left(1081 \mathrm{~cm}^{-1}\right)$.

O índice I1630/I2920 informa sobre o grau de insaturação/ saturação (intensidade das vibrações de grupos $\mathrm{C}=\mathrm{O}$ e $\mathrm{C}=\mathrm{C}$ em relação à intensidade de grupos C-H) da fração analisada. Para a fração de $\mathrm{AH}$ os valores se situaram entre 0,55 e 0,75 (Tabela 3). Em ambas profundidades analisadas, os menores valores da razão I1630/I2920 foram observados no sistema APC, corroborando os dados de OLENDZKI (2006) para AH de planossolos sob cultivo de arroz. O maior valor desta razão encontrada para os AH sob APD em comparação ao de APC indica uma menor proporção de grupos alifáticos, o que pode ser indicativo de um menor grau de humificação de AH no sistema conservacionista. O enriquecimento relativo de grupos alifáticos é atribuído

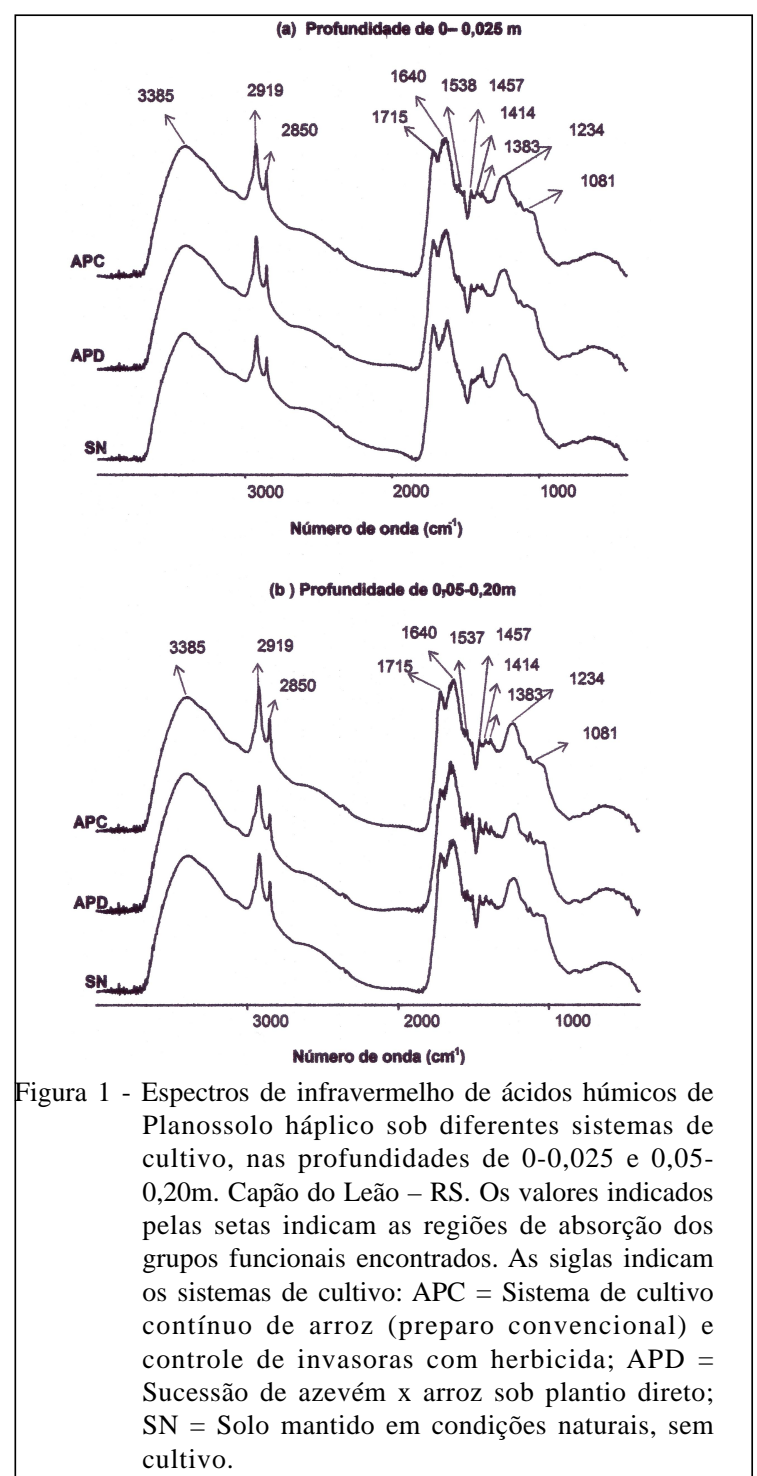

Ciência Rural, v.38, n.6, set, 2008. 
Tabela 3 - Índice $\mathrm{I}_{1630} / \mathrm{I}_{2920}$, composição elementar, relação C/N e índice de polaridade ((O+N)/C) dos ácidos húmicos extraídos das camadas de 0-0,025 e 0,05-0,20m de um Planossolo háplico, submetido a diferentes sistemas de cultivo, Capão do Leão - RS. Média de duas repetições.

\begin{tabular}{|c|c|c|c|c|c|c|c|c|}
\hline Sistema de manejo & $\mathrm{I}_{1630} / \mathrm{I}_{2920}(\mathrm{FTIR})$ & Cinzas (\%) & C (\%) & $\mathrm{H}(\%)$ & $\mathrm{N}(\%)$ & $\mathrm{O}(\%)$ & $\mathrm{C} / \mathrm{N}$ & $(\mathrm{O}+\mathrm{N}) / \mathrm{C}$ \\
\hline & \multicolumn{8}{|c|}{ 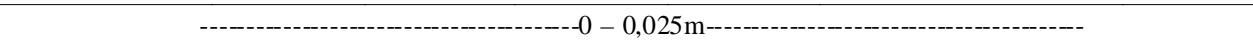 } \\
\hline APC & 0,57 & 0,37 & 55 & 3,8 & 3,6 & 37 & 15,1 & 0,56 \\
\hline APD & 0,74 & zero & 52 & 4,8 & 3,3 & 39 & 15,7 & 0,62 \\
\hline \multirow[t]{2}{*}{$\mathrm{SN}$} & 0,62 & zero & 53 & 5,0 & 3,5 & 37 & 15,1 & 0,58 \\
\hline & & & & $--0,05$ & $1---\cdot$ & 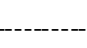 & ----- & \\
\hline APC & 0,55 & zero & 53 & 4,4 & 3,5 & 38 & 15,2 & 0,78 \\
\hline APD & 0,75 & zero & 48 & 3,6 & 3,3 & 44 & 14,6 & 0,99 \\
\hline SN & 0,73 & 3,93 & 56 & 5,3 & 3,7 & 34 & 15,2 & 0,67 \\
\hline
\end{tabular}

APC = Sistema de cultivo contínuo de arroz (preparo convencional) e controle de invasoras com herbicida; APD = Sucessão de azevém $\mathrm{x}$ arroz sob plantio direto; $\mathrm{SN}=$ Solo mantido em condições naturais, sem cultivo.

ao consumo preferencial de estruturas O-alquil (DICK et al, 2005), o que estaria sendo favorecido no sistema APC, devido ao revolvimento.

Os valores de $\mathrm{C}, \mathrm{H}, \mathrm{N}$ e $\mathrm{O}$ dos $\mathrm{AH}$ foram similares entre os tratamentos nas duas camadas analisadas (0-0,025m e 0,05-0,20m) (Tabela 3), e são semelhantes aos valores observados para AH de solos hidromórficos (DICK \& MARTINAZZO, 2006). Os teores de $\mathrm{N}$ são inferiores aos usualmente observados para solos bem drenados, resultando numa relação $\mathrm{C} / \mathrm{N}$ comparativamente mais elevada (Tabela 3). Os AH do sistema APD apresentaram índice de polaridade superior ao dos AH de APC em ambas as profundidades. A menor polaridade de AH sob APC é decorrente provavelmente da decomposição preferencial de grupos oxigenados e nitrogenados, ocorrendo um enriquecimento de grupos recalcitrantes alifáticos, conforme já indicado pelo índice I1630/I2920. Em ambos os sistemas de cultivo, a polaridade dos AH aumenta com a profundidade, sugerindo a migração de compostos húmicos ácidos em profundidade. Os AH do sistema SN apresentaram comportamento diferenciado do índice de FTIR e de polaridade em relação aos AH sob sistemas de cultivo. Provavelmente, a diferente vegetação afeta a qualidade dos $\mathrm{AH}$, limitando assim a comparação entre os três tratamentos analisados.

\section{CONCLUSÕES}

O sistema plantio direto preserva os teores de carbono orgânico total e em todas as frações húmicas. O sistema de manejo com preparo convencional do solo, provoca perdas de carbono orgânico total, sendo que essas perdas se verificam em todas as frações húmicas. Na camada de 0 a 0,025m, o sistema plantio direto tende a preservar a matéria orgânica, propiciando a ocorrência de substâncias húmicas de menor grau de decomposição, mais polares e contendo menores proporções de estruturas alifáticas em comparação ao sistema de preparo convencional.

\section{REFERÊNCIAS}

ALMEIDA, J.A. et al. Propriedades químicas de um Cambissolo Húmico sob preparo convencional e semeadura direta após seis anos de cultivo. Revista Brasileira de Ciência do Solo, v.29. n.3. p.437-445, 2005.

AZAMBUJA, I.H.V. et al. Aspectos sócio-econômicos da produção do arroz. In: GOMES, A.S.; MAGALHÃES JÚNIOR, A.M. Arroz irrigado no sul do Brasil. Brasília, DF: Embrapa Informação Tecnológica, 2004. p.23-44.

BAYER, C.; BERTOL, I. Características químicas de um Cambissolo húmico afetadas por sistemas de preparo, com ênfase a matéria orgânica. Revista Brasileira de Ciência do Solo, v.23, p.687-694, 1999.

CHEFETZ, B. et al. Chemical and biological characterization of organic matter during composting of municipal solid waste. Journal of Enviromental Quality, v.25. p.776-785, 1996.

CHEN Y. et al. Information provided on humic substances by E4/E6 ratios. Soil Science of America Journal, v.41, p.352358, 1977.

COSTA, F. de S. et al. Aumento de matéria orgânica num Latossolo bruno em plantio direto. Ciência Rural, v.34, n.2, p.587-589, 2004.

DICK, D. P. et al. Caracterização de produtos de decomposição de lodos orgânicos em diferentes tempos e meios de digestão. Revista Brasileira de Ciência do Solo, v.21, n.1, p.1-8, 1997.

DICK, D.P. et al. Caracterização de substâncias húmicas extraídas de solos e de lodo orgânico. Revista Brasileira de Ciência do Solo, v.22, 603-611, 1998. 
DICK, D.P. et al. Characteristics of soil organic matter of different Brazilian Ferralsols under native vegetation as a function of soil depth. Geoderma, v.124, p.319-333, 2005.

DICK, D.P.; MARTINAZZO, R. Matéria orgânica em ambientes terrestres e aquáticos: compartimentos, composição e reações. In: POLETO, C.; MERTEN, G.H. Qualidade dos sedimentos. Porto Alegre: Associação Brasileira de Recursos Hídricos, 2006. p.141-179.

EMBRAPA - Centro Nacional de Pesquisa de Solos. Sistema Brasileiro de Classificação de Solos. Brasília: EMBRAPA - Produção de informação, Rio de Janeiro: EMBRAPA - Solos, 1999. 412p.

KIRK, G.J.D; OLK, D.C. Carbon and nitrogen dynamics in flooded soils. Los Baños, Philippines: International Rice Research Institute, 2000. 188p.

MAIE, N. et al. Origin and properties of humus in the subsoil of irrigated rice paddies-III. Changes in binding type of humus in submerced plow layer soil. Soil Science and Plant Nutrition, v.44, n.3, p.331-345, 1998.

OLENDZKI, R.N. Caracterização das interações de substâncias húmicas com a matéria inorgânica em solos de cultivo de arroz irrigado: contribuição à aquisição de dados essenciais para avaliação global do seqüestro de carbono no solo. 2006. 134f. Tese (Doutorado em Química - Setor de Ciências Exatas) - Universidade Federal do Paraná, Curitiba.
OLIVEIRA, C.F. de. Censo da lavoura de arroz irrigado do Rio Grande do Sul - safra 2004/5. Porto Alegre: IRGA - Política Setorial, 2006. 122p.

OLK, D.C. et al. Decrease in humification of organic matter with intensified lowland rice cropping: A wet chemical and spectroscopic investigation. Soil Science of American Journal, v.64, p.1337-1347, 2000.

PAIM, S. et al. Caracterization of fungal melanins and soil humic acids by chemical analysis and infrared spectroscopy. Biology and Fertility of Soils, v.10, p.72-76, 1990.

PINTO, L.F.S. et al. Solos de várzea do sul do Brasil cultivados com arroz irrigado. In: GOMES, A.S.; MAGALHÃES JR., A.M. Arroz irrigado no sul do Brasil. Brasília, DF: Embrapa Informação Tecnológica, 2004. p.75-95

SANTOS, V.B. dos et al. Biomassa, atividade microbiana e teores de carbono e nitrogênio totais de um planossolo sob diferentes sistemas de manejo. Revista Brasileira de Agrociência, v.10, n.3, p.333-338, 2004.

TAN, K.H. Humic Matter in soil and the environment. Madison Avenue, New York: Marcel Decker, 2003. 385p.

TORRENTS, A. et al. Influence of the polarity of organic matter on the sorption of acetamide pesticides. Journal of Agricultural Food and Chemistry, v.45, p.3320-3325, 1997. 